\title{
Méthode d'évaluation des risques des produits
}

\author{
A method for evaluating the products risks \\ par Pr. Ph. Hartemann \\ Laboratoire d'Hygiène et de Recherche en Santé Publique, Faculté de Médecine, \\ Université Henri Poincaré Nancy
}

Risk evaluation concerning health and environment products used qualitative after semi-quantitative information; now it uses a well defined and rigorous methodology and quantitative information. It aims at elaborating rules in order to protect a population on the basis of a quantified residual risk, which is considered as acceptable. But it remains a lot of uncertainties which are at the origin of differences between acceptable maximum concentrations determined by different ways.

\section{I $\square$ INTRODUCTION}

Protéger l'environnement et protéger la santé sont deux préoccupations majeures et difficilement dissociables exprimées par nos concitoyens dont le niveau d'inquiétude oscille en fonction du battage médiatique fait autour de telle ou telle affaire (dioxines, lait et fumées d'usines d'incinération ; algues toxiques et coquillages ; nitrates, agriculture et eau de boisson pour citer quelques exemples de ces derniers mois).

Il n'est pas contestable que de nombreux dangers à court ou long terme sont liés à l'exposition aiguë ou chronique à des facteurs environnementaux, parmi lesquels les produits chimiques sont les plus " ciblés" par l'opinion et les média. Il existe donc une demande de plus en plus forte pour des mesures permettant de limiter les émissions de contaminants et leur niveau dans les différents milieux (air, eau, sols, aliments), la concentration zéro apparaissant pour les " intégristes " comme le niveau nécessaire, quel qu'en soit le coût.

Puisque la santé de la population est mise en avant pour obtenir cette réduction de la contamination, il apparaît donc indispensable de s'assurer que les actions induites trouvent une pleine justification en terme de réduction des risques (probabilité de trouble) pour la santé. Malheureusement cette démarche est difficile car la quantification du lien entre polluant environnemental et état de santé est complexe : estimation de l'exposition et de la dose pour chaque individu, manque de puissance statistique des études épidémiologiques quand le risque à évaluer est faible, validité de l'extrapolation des résultats toxicologiques de l'animal à l'homme, synergie éventuelle des effets de multiples contaminants lors d'une exposition obligatoirement simultanée. Le hiatus est donc important entre la difficulté de la connaissance scienti- fique et l'attente des populations et des décideurs chauffés à blanc par les média. Ceci conduit à " prendre des décisions dures avec des connaissances molles " et le principe de précaution, qui réintroduit le doute dans une réflexion avant prise de décision, joue à plein. Il doit induire une démarche d'évaluation quantitative des risques à la place de l'évaluation qualititative ou semi-quantitative qui prévalait jusqu'à maintenant, avec le passage de l'âge de la prévention à celui de la précaution.

\section{II —U'EST-CE QUE L'ÉVALUATION DES RISQUES ?}

Depuis Hippocrate montrant la relation entre paludisme et marais, il y a de nombreux exemples d'une description purement qualitative de la relation santé-environnement. Après la deuxième guerre mondiale, les progrès de la toxicologie et de l'épidémiologie permettent une approche semi-quantitative qui prend appui sur l'expérimentation animale et les observations chez l'homme, mais limitées à la toxicité aiguë ou busaiguë. Sont introduites les notions de valeurs limites d'exposition professionnelle, de dose journalière admissible, reposant sur l'extrapolation linéaire du risque avec des coefficients de sécurité selon le niveau d'obtention des données. C'est à partir du risque cancérogène et de l'expérience de l'industrie nucléaire que la réflexion mise en œuvre par diverses agences américaines débouchera sur l'évaluation quantitative des risques. Les concepts seront formalisés en 1983 par le National Research Council et l'Académie des Sciences des USA, puis mis à jour en 1994 : “ Utilisation de données factuelles pour définir les effets sur la santé de l'exposition d'individus ou de populations à des matériaux ou des situations dangereuses ". 
La démarche vise à élaborer une réglementation pour protéger l'ensemble de la population sur sa durée de vie (70 ans) en prenant comme base un risque résiduel quantifié, considéré comme acceptable : par exemple un décès supplémentaire par cancer pour un million d'habitants. L'extrapolation à partir des données expérimentales ou épidémiologiques ne se fait plus par avis d'experts mais par application d'une méthodologie scientifique clairement explicitée.

Celle-ci comprend différentes phases en un processus itératif :

- la production de données $=$ identification des dangers

- connaissance des molécules présentes ou susceptibles d'être formées,

- recherche de l'existence d'une relation dose-effet, au niveau d'un individu (animal voire humain) à partir des données de la littérature ou d'expérimentations,

- recherche de l'existence d'une relation dose-réponse, au niveau d'une population (données épidémiologiques et/ou cliniques de la littérature) ;

- l'évaluation des risques (risk assessment)

- évaluation des émissions.

- évaluation des expositions,

- évaluation des conséquences (quantification de la relation entre expositions et conséquences pour la santé et l'environnement),

- estimation des risques (estimation de la probabilité, du moment de la survenue, de la nature et de la grandeur des conséquences défavorables) prenant en compte les relations dose-effet et dose-réponse :

- la gestion des risques (risk management)

- génération d'options,

- évaluation des options,

- sélection des options,

- mise en œuvre et exécution,

- évaluation.

\section{III — L'ÉTAPE DE LA RELATION ENTRE LA DOSE ET L'EFFET}

Il existe un débat sur l'existence ou non d'un seuil pour les substances cancérigènes. L'approche toxicologique (effet sans seuil) utilise un certain nombre de facteurs de sécurité qui permettent de passer de l'expérimentation sur l'animal à l'extrapolation à l'homme (1000 si données humaines concluantes, 100 si données animales à long terme mais données humaines incertaines, 10 si données animales incertaines sans données humaines, dans le cas de substances cancérigènes), 10 si passage de l'animal à l'homme, 10 pour la susceptibilité individuelle etc.

Si l'on utilise la notion de seuil, le modèle employé à partir de données expérimentales identiques conduit à obtenir des effets attendus aux faibles doses variant de façon considérable. L'exemple de l'effet cancérigène de la saccharine sur la vessie montre que l'on passe de 840 cas pour 50 millions d'habitants avec le modèle " one hit " extrapolation aux faibles doses et ajustement sur la surface corporelle pour le passage de l'animal à l'homme, à 70000 cas pour 50 millions d'habitants avec le module " multi hit " et un ajustement sur le poids corporel. Dans d'autres cas on peut arriver à une variation des cas attendus de l'ordre de 107 . Ces incertitudes des toxicologues traduisent bien la difficulté du travail pour présenter des scénarios crédibles.

\section{IV — L'ÉTAPE DE LA MESURE DES EXPOSI- TIONS DANS LES POPULATIONS}

C'est celle dans laquelle notre laboratoire s'est spécialisé : elle peut aller de la simple mesure environnementale à celle d'indicateurs biologiques d'exposition au sein de la population cible. Il convient de faire au départ un certain nombre d'hypothèses, validées au niveau international quant aux caractéristiques physiologiques des populations (masse corporelle, volumes d'air respirés, d'eau consommée, d'aliments et de poussières ingérés, etc.) à chaque âge de la vie. Pour proposer une valeur paramétrique pour une norme environnementale, on part de la dose journalière acceptable ou des modèles pour ce qui concerne les substances cancérigènes, et on estime ce qui est apporté par les différents milieux (air, eau, alimentation, etc.). Différents scénarios sont construits en faisant varier les apports dans les différents milieux en fonction des données des mesures effectuées en leur sein dans diverses situations. On retient ensuite le scénario le plus plausible ou le plus critique pour proposer une valeur pour l'apport du produit dans le milieu à normaliser.

Ainsi l'évolution des recommandations de l'OMS sur le plomb dans l'eau passant de $50 \mu \mathrm{g} / \mathrm{l}$ à $10 \mu \mathrm{g} / \mathrm{l}$ repose sur une diminution de moitié de la valeur de l'apport hebdomadaire acceptable chez l'enfant, fondée sur la demande des toxicologues de ne pas accepter d'accumulation de plomb dans l'organisme (en dehors de tout effet connu). Un apport de $5 \mu \mathrm{g}$ de plomb par jour chez un nourrisson introduit dans un scénario très "dur" (fabrication des biberons avec de l'eau du robinet, $50 \%$ des apports liés à cette source) conduit à une concentration dans l'eau de $11,7 \mu \mathrm{g} / \mathrm{l}$ à ne pas dépasser. Dans le même temps les CMA dans le lait ou les jus de fruit ont des valeurs supérieures ! On voit donc que cette étape est très importante et peut conduire à la fixation de CMA aux conséquences économiques très lourdes.

\section{V — LE PROBLÈME DES PRODUITS PHYTO- SANITAIRES ET DES NITRATES}

Les bases de la fixation des recommandations de l'OMS relatives aux paramètres chimiques reposent sur deux principes différents, selon qu'elles s'adressent à des molécules à effet de type déterministe ou probabiliste.

Dans le premier cas, ces molécules ont un effet sur la santé qui est bénéfique ou neutre à faible dose, mais dont l'importance, croissant avec la dose, conduit à partir d'un certain niveau d'ingestion, à observer un effet pathologique. La gravité de celui-ci sera fonction de la dose absorbée (relation dose-effet) et au sein d'une population hétérogène, de sensibilités différentes, la relation dose-réponse sera fonction du niveau entraînant la pathologie chez les individus les plus sensibles (ex. nourrissons). Le principe est alors d'éviter l'apparition du moindre cas de pathologie et de fixer des limites de concentration avec une marge de sécurité telle que, même sur la base des sujets les plus sensibles, les apports cumulés sur une vie entière ne puissent avoir la moindre conséquence néfaste : conception de protection "absolue". C'est ce schéma qui a été utilisé pour les nitrates qui n'ont par eux-mêmes aucun effet toxique, mais dont la transformation en nitrites peut entraîner un effet sur la santé. La valeur de $50 \mathrm{mg} / \mathrm{l}$ apparaît très contraignante mais son dépassement n'entraîne aucun risque réel pour la quasi-totalité de la population. 
Dans le deuxième cas, les molécules à effet de type stochastique se caractérisent par l'absence d'une relation doseeffet, mais leur présence augmente la probabilité d'apparition d'une pathologie (ex. cancer, leucémie, anomalie génétique, etc.) existant par ailleurs au sein d'une population non exposée. On ne peut plus utiliser le concept d'absence d'apparition de la maladie et l'OMS fixe alors un niveau de fréquence "acceptable" à un cas par 100000 personnes exposées durant leur vie entière au sein de la population pour déterminer la concentration maximale acceptable dans les intrants (eau-aliments, etc.).

Au niveau des directives européennes, les valeurs de référence reprennent pour partie les recommandations de l'OMS, en acceptant donc ces bases de raisonnement toxicologiques, telles quelles pour les molécules à effet déterministe (ex. plomb), ou en "sévérisant" la fréquence acceptable à $10^{-6}$ (au lieu de $10^{-5}$ ) pour les molécules à effet probabiliste. Mais pour les produits phytosanitaires, l'Union européenne ne reconnaît pas les bases toxicologiques ; elle retient un niveau uniforme $(0,1 \mu \mathrm{g} / \mathrm{l})$ pour n'importe quelle molécule sur la base d'un souhait "écologique". D'autres pays ont en revanche fondé leur législation sur les bases toxicologiques pour ce qui concerne les pesticides ou les sous-produits de désinfection de l'eau par le chlore. Ainsi les recommandations de l'OMS (au risque $10^{-5}$ ) portent sur 19 produits phytosanitaires individuels ou groupes d'isomères avec une gamme de valeurs paramétriques allant de 0,01 à $175 \mu \mathrm{g} / \mathrm{l}$, la législation des Etats-Unis retient 18 composés ou groupes d'isomères de 0,05 à $400 \mu \mathrm{g} / \mathrm{l}$, celle du Canada cite $37 \mathrm{com}$ posés ou groupes d'isomères de 0,7 à $900 \mu \mathrm{g} / \mathrm{l}$, (et un total de $100 \mu \mathrm{g} / \mathrm{l}$ ), celle d'Australie énumère 101 composés avec des CMA de 0,4 à $3000 \mu \mathrm{g} / \mathrm{l}$. Le décret français $89-3$ prend en compte la directive européenne d'une CMA de $0,1 \mu \mathrm{g} / \mathrm{l}$ par substance individualisée et de $0,5 \mu \mathrm{g} / \mathrm{l}$ pour le total des produits phytosanitaires (comment le mesurer (?!) mais l'a complété par des CMA de $0,01 \mu \mathrm{g} / \mathrm{l}$ pour I'hexachlorobenzène et de $0,03 \mu \mathrm{g} / \mathrm{l}$ pour l'heptachlore, l'aldrine et la dieldrine afin de respecter le niveau de risque acceptable à $10^{-6}$. L'Américain du nord ou l'Australien sont-ils plus mal protégés pour autant? La nouvelle directive européenne n'a pas varié sur ce point et nous continuerons donc à "traîner" ce paramètre comme un boulet, avec une CMA sans aucun rapport avec une évaluation quantitative du risque.

\section{VI — CONCLUSION}

Si l'on prend en compte le fait qu'avec les nouvelles réglementations, l'industrie chimique est tenue de réévaluer 100000 substances et doit garantir, à terme, que les nouveaux produits ne sont pas nocifs pour l'environnement, on voit qu'il y a là un énorme marché pour l'écotoxicologie et l'évaluation des risque pour l'environnement et pour la santé humaine. Le rythme actuel étant de l'ordre de 300 dossiers par an, il y a de l'espoir pour nos descendants ! Dans un tel contexte (presque) tous les coups semblent permis. L'expert (ou présumé tel) a donc un certain nombre de devoirs :

- celui de "parler vrai" et donc de se méfier des effets d'annonce en renonçant dans notre monde où la norme est "publish or perish" à la tentation de s'assurer une priorité et/ou une célébrité en annonçant trop tôt un résultat "spectaculaire". Il doit être scrupuleux quant à la portée des extrapolations,

- celui d'être courageux, car certains des résultats seront désagréables, voire scandaleux, pour l'un ou l'autre camp,

- celui d'être humble, compte tenu de toutes les incertitudes énumérées précédemment, de façon à résister au "terrorisme pansanitaire".

Dans ce contexte il semble apparaître que ces mesures de contrôle de qualité de l'eau-aliment conduisent à avoir un niveau de sécurité très élevé sur le plan de la chimie (et moindre sur celui de la microbiologie). En effet, les bases de fixation des CMA pour les produits chimiques prennent en compte au minimum une limitation du risque de pathologie à 1 cas sur un million de consommateurs d'eau pendant leur vie entière et, hélas, dans la situation présente la probabilité de développer un cancer durant sa vie est de l'ordre de 5.10-1 (une "chance" sur deux !) pour les Français. Le rôle de l'eau est donc pour le moins très faible, sinon négligeable à côté d'autres dangers bien connus (ex. tabac). Il est donc quasi impossible de mettre en évidence épidémiologiquement un effet néfaste mesurable sur la santé des populations par rapport au bruit de fond, sauf dans des situations de dépassement très important de la CMA.

\section{BIBLIOGRAPHIE}

[1] National Research Council (National Academy of Sciences). Risk assessment in the federal government : managing the process. National Academy Press, Washington DC. 1983.

[2] US Environmental Protection Agency. Risk assessment and management : framework for decision making. US - EPA, December 1984.

[3] US Environmental Protection Agency. The risk assessment guidelines of 1986. US - EPA, August 1987

[4] Déclaration de Rio en 1992. (introduisant le principe de précaution).

[5] Commission des Communautés Européennes. Directive 93/67/CEE : principes d'évaluation des risques pour l'homme et pour l'environnement des substances notifiées conformément à la directive 67/548/CEE. J.O. CEE, 20 juillet 1993.

[6] COVELLO V.T. MERKHOFFER M.W. Risk assessment methods approaches for assessing health and environmental risks. Plenum Press, 1994. 